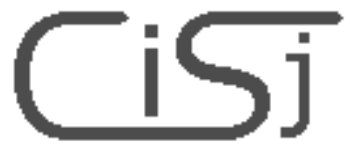

\title{
SYMBOLIC MODELS OF THE PULSE ENERGY CONVERSION SYSTEMS DYNAMICS
}

\author{
Koschinsky S.L., Sholonik A.P., Ustinov P.S. \\ Radioelectronics Department, \\ State Technical University of Orel, \\ 29, Naugorskoye Shosse, 302020 Orel, Russia, \\ tel: 70862 419879, fax: 70862 416684, \\ e-mail: kipra@ ostu.ru
}

\begin{abstract}
The derivation of developed symbolic models of dynamics of voltage mode controlled buck converter operated both in discontinuous and continuous conducting modes is given.
\end{abstract}

Keywords: - Symbolic model, symbolic characteristic, nonlinear dynamics, buck converter.

\section{INTRODUCTION}

Pulse energy conversion systems (PECS) are widely used in modern energetics. PECS are complex, essentially nonlinear systems with complicated dynamics. There is a great number of undesirable stationary modes except synchronous one could be possibly realized in PECS. The appearance of such mode will lead to essential increase of the current and voltage ripples in PECS elements as well as to essential degradation of the output energy quality. To exclude these undesirable modes the research of PECS dynamics should be realized at PECS design stage.

The mathematical model of PECS as an essentially nonlinear system is commonly represented as a system of ordinary differential equations

$$
\frac{d \mathbf{X}}{d t}=\mathbf{G}(\mathbf{X}, \mathbf{P}, t),
$$

where $\mathbf{G}(\mathbf{X}, \mathbf{P}, t)$ is the piecewise smooth vector function; $\mathbf{X}=\left\{x_{1}, x_{2}, \ldots, x_{n}\right\}$ is system state vector; $\mathbf{P}=\left\{p_{1}, p_{2}, \ldots, p_{s}\right\}$ is system parameters vector.

Let $\mathbf{P}\left(p_{1}, p_{2}, \ldots, p_{s}\right)$ to be the space of the system parameters. There are different trajectories defined in system (1) extended state space $(\mathbf{X}, t)$. The specific structure of state space $(\mathbf{X}, t)$ can be defined to any point $p \in \mathbf{P}$ and consists of a number and placement of stationary trajectories. The space $\mathbf{P}$ is divided into regions $\mathrm{P}_{k}$ that are corresponded to topologically equivalent structure of the extended state space $(\mathbf{X}, \mathrm{t})$. When system parameters $\mathbf{P}$ are bifurcational the topological structure of space $(\mathbf{X}, t)$ changes. The geometrical place of the bifurcational points defines some bifurcation surface in space $\mathbf{P}$. Thus the research of the system (1) dynamics can be considered as the composition of the bifurcation boundaries for $\mathrm{P}_{k}$ regions and analysis of the specific structure of the state space for these regions [1].

The linguistic description of PECS dynamics that is used at present time seems to be very clumsy. This disadvantage makes inconvenient the detailed research of PECS dynamics. An effective tool to provide PECS dynamics analysis is the usage of symbolic description of its dynamic modes [1, 2]. For example, symbolic models of dynamic modes are efficiently used for analysis of mechanic systems with dry friction and vibration shock [1]. Both the considered in [1] mechanic systems as well as PECS have similar mathematical models with discontinuous right-hand part (1). The symbolic model of dynamic mode is the sequence of symbols corresponded to the sequence of intervals of (1) structure constancy where considered dynamic mode is defined. PECS symbolic models that are composed on the base of description [1] with additional rules and signs reveal types of PECS bifurcation boundaries and simplify the analysis of PECS dynamics.

This paper deals with derivation of developed symbolic models of dynamics of voltage mode controlled buck converter operated both in discontinuous and continuous conducting modes. 


\section{MATHEMATICAL MODEL OF THE BUCK CONVERTER}

Equivalent scheme of the buck converter is shown in Fig. 1.

Mathematical model of the power unit of the buck converter corresponding to equivalent scheme (Fig. 1) has the form:

$$
\begin{aligned}
& \frac{d \mathbf{X}(\gamma)}{d \gamma}=T\left(\mathbf{A} \cdot K_{F 1}+\mathbf{A}_{1} \cdot\left(1-K_{F 1}\right)\right) \mathbf{X}(\gamma)+ \\
& +T \cdot \mathbf{B} \cdot K_{F 0}
\end{aligned}
$$

where $\mathbf{X}(\gamma)=\left(\begin{array}{c}i(\gamma) \\ u(\gamma)\end{array}\right)$ is state variables vector; $i(\gamma)$ is the inductor current; $u(\gamma)$ is the capacitor voltage; $\gamma=\frac{t}{T}, \gamma \in[0,1]$ is the relative pulse duration; $T$ is the PWM clock instant;

$$
\begin{aligned}
& \mathbf{A}=\left(\begin{array}{cc}
-\frac{1}{L}\left(R_{1}+\frac{R_{2} \cdot R_{3}}{R_{2}+R_{3}}\right) & -\frac{R_{3}}{L \cdot\left(R_{2}+R_{3}\right)} \\
\frac{R_{3}}{C \cdot\left(R_{2}+R_{3}\right)} & -\frac{1}{C \cdot\left(R_{2}+R_{3}\right)}
\end{array}\right), \\
& \mathbf{A}_{1}=\left(\begin{array}{cc}
0 & 0 \\
0 & -\frac{1}{C \cdot\left(R_{2}+R_{3}\right)}
\end{array}\right), \mathbf{B}=\left(\begin{array}{c}
\frac{E}{L} \\
0
\end{array}\right) \text { are the }
\end{aligned}
$$

square matrices and column vector determined by element values of equivalent scheme in Fig.1.

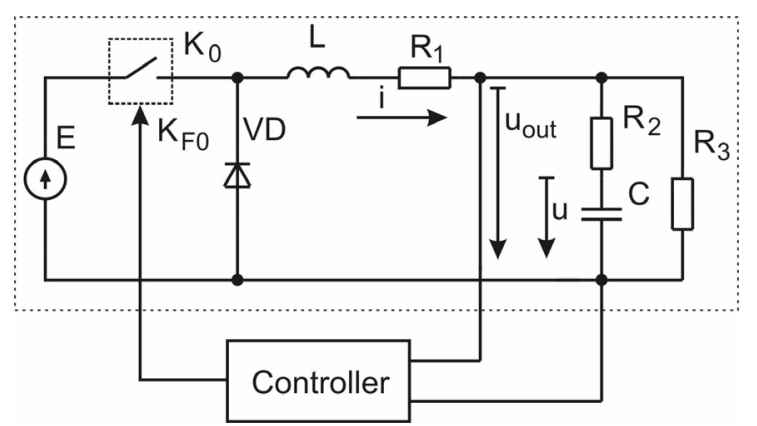

Fig. 1 - Equivalent scheme of the buck converter.

Controller realizes trailing edge pulse-width modulation and proportional control of the output voltage. The pulse function $K_{F 0}$ in model (2) is calculated according to the algorithm

$$
K_{F 0}= \begin{cases}1, & 0<\gamma \leq \gamma_{0} \\ 0, & \gamma_{0}<\gamma \leq 1\end{cases}
$$

where $\gamma_{0}$ is the switch moment corresponding to transition of $\mathrm{K}_{0}$ switch to non-conducting state and diode VD to conducting state.
Value $\gamma_{0} \in[0,1]$ is evaluated as the least root of the equation which determines a surface where the right-hand part of (2) has discontinuities

$$
\begin{aligned}
& \zeta_{0}(\mathbf{X}(\gamma), \gamma)=\alpha \cdot\left(U_{r e f}-\beta \cdot \mathbf{C}_{\mathbf{0}} \cdot \mathbf{X}(\gamma)\right)- \\
& -U_{0} \cdot \gamma=0,
\end{aligned}
$$

where $\alpha$ is the proportional gain; $\beta$ is the gain of the output voltage sensor; $U_{r e f}$ is the reference voltage; $U_{0}$ is the sawtooth voltage amplitude; $\mathbf{C}_{\mathbf{0}}$ is the row vector which sets up a correspondence between $\mathbf{X}(\gamma)$ and the voltage value of the controller input $\left(\mathrm{u}_{\mathrm{out}}\right)$.

The pulse function $K_{F 1}$ in model (2) is calculated according to the algorithm

$$
K_{F 1}= \begin{cases}1, & \gamma_{0}<\gamma \leq \gamma_{1} \\ 0, & \gamma_{1}<\gamma \leq 1\end{cases}
$$

where $\gamma_{1}$ is the switch moment corresponding to transition of diode VD to non-conducting state.

Value $\gamma_{1} \in\left[\gamma_{0}, 1\right]$ is evaluated as the least root of the equation

$$
\zeta_{1}(\mathbf{X}(\gamma))=\mathbf{C}_{1} \cdot \mathbf{X}(\gamma)=0, \mathbf{C}_{1}=\left(\begin{array}{ll}
1 & 0
\end{array}\right)
$$

\section{ELEMENTS OF THE GEOMETRICAL STRUCTURE OF THE BUCK CONVERTER STATE SPACE}

Let $\mathbf{G}$ to be considered state space of buck converter model (2-6). There are three pairs of the values of pulse functions $K_{F 0}, K_{F 1}-\left(K_{F 0}=1\right.$; $\left.K_{F l}=0\right),\left(K_{F 0}=0 ; K_{F l}=1\right),\left(K_{F 0}=0 ; K_{F 1}=0\right)$ which may be realized physically in PECS. There are three states of the system (1) respectively to every pair of pulse functions and thus we can determine domains $\mathbf{G}_{\mathbf{1}}, \mathbf{G}_{\mathbf{2}}, \mathbf{G}_{\mathbf{3}}$ in $\mathbf{G}$ space for each system (1) state. The transition of the state point in these domains is determined by (2) on the intervals of the structure constancy. Surfaces $\mathbf{S}_{\mathbf{1}}, \mathbf{S}_{\mathbf{2}}, \mathbf{S}_{\mathbf{3}}$ are the boundaries of the $\mathbf{G}_{\mathbf{1}}, \mathbf{G}_{\mathbf{2}}, \mathbf{G}_{\mathbf{3}}$ domains (Fig. 2):

$$
\begin{gathered}
\mathbf{S}_{\mathbf{1}} \equiv \zeta_{0}(\mathbf{X}(\gamma), \gamma)=0 ; \mathbf{S}_{\mathbf{2}} \equiv \gamma=0 ; \\
\mathbf{S}_{\mathbf{3}} \equiv \mathbf{G}_{\mathbf{3}} \equiv \zeta_{1}(\mathbf{X}(\gamma))=0 .
\end{gathered}
$$

Surface $\mathbf{S}_{\mathbf{2}}$ is divided in two components: 


$$
\begin{aligned}
& \mathbf{S}_{\mathbf{2 ( 1 )}}, \beta \cdot \mathbf{C}_{0} \mathbf{X}(\gamma)<\left.U_{r e f}\right|_{\gamma=0} ; \\
& \mathbf{S}_{\mathbf{2 ( 2 )}}, \beta \cdot \mathbf{C}_{0} \mathbf{X}(\gamma) \geq\left. U_{r e f}\right|_{\gamma=0}
\end{aligned}
$$

Suppose that intersection of surfaces $\mathbf{S}_{\mathbf{2}(\mathbf{2})}$ and $\mathbf{S}_{\mathbf{3}}$ belongs to the surface $\mathbf{S}_{\mathbf{3}}$, and intersection of surfaces $\mathbf{S}_{\mathbf{2 ( 1 )}}$ and $\mathbf{S}_{\mathbf{3}}$ belongs to the surface $\mathbf{S}_{\mathbf{2 ( 1 )}}$. The transition of the state point in the domains $\mathbf{G}_{\mathbf{1}}, \mathbf{G}_{\mathbf{2}}$, $\mathbf{G}_{\mathbf{3}}$ between surfaces $\mathbf{S}_{\mathbf{1}}, \mathbf{S}_{\mathbf{2 ( 1 )}}, \quad \mathbf{S}_{\mathbf{2 ( 2 )}}, \mathbf{S}_{\mathbf{3}}(7,8)$ corresponds to change of the buck converter state.

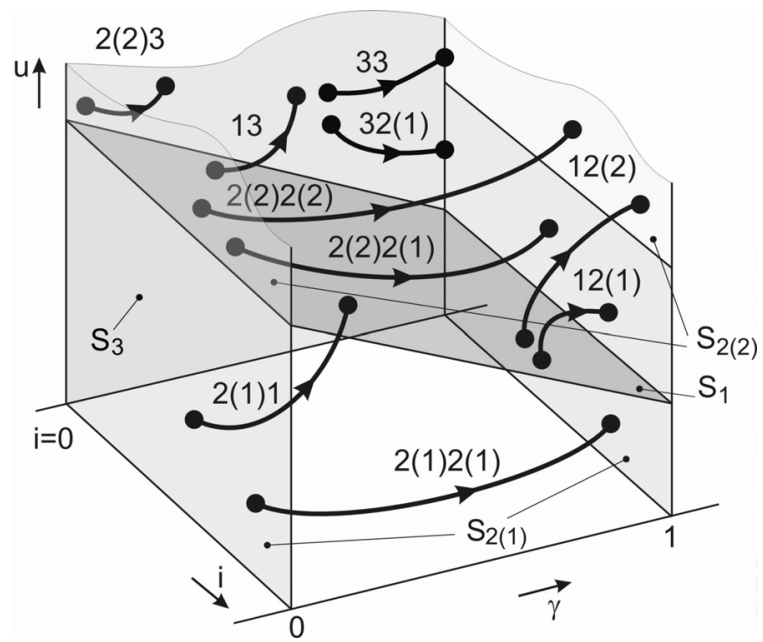

Fig. 2 - State space of the buck converter.

During this transition state point draws some trajectory. There are parts of the trajectories which are placed between two surfaces on which system structure doesn't change. Such parts of the trajectory we call the simplest parts. If we take into consideration above-mentioned surfaces there are 16 simplest parts which have quantitative differences. If the start point of the simplest part belongs to the surface $i$ and the end point belongs to the surface $j$ we denote such part as aggregative symbol $i j$. For example, the start point of the part belongs to the surface $\mathbf{S}_{\mathbf{1}}$ and the end point belongs to the surface $\mathbf{S}_{\mathbf{2 ( 1 )}}$. We denote this part as 12(1).

From 16 simplest parts we mark those, which satisfy the following conditions:

1) the parts may be realized physically;

2) there is a satisfaction of one of the next conditions:

2.1) system structure changes at the end point of the part; $\mathbf{S}_{2}$.

2.2) end point of the part belongs to the surface

10 from 16 possible simplest parts satisfy these conditions. There are 12(1); 12(2); 13; 2(1)1; 2(1)2(1); 2(2)2(1); 2(2)2(2); 2(2)3; 32(1); 33.

The simplest parts $32(1), 33$ correspond to sliding motions [3].
Under sliding motion we consider transition of the state point in the subspace which dimension is less than dimension of the basic state space, i.e. transition on the surface where right-hand part of the system of equations (2) has a discontinuity. If there is sliding part in the trajectory of some mode we call it sliding mode or for this model - discontinuous conducting mode.

It is necessary to add a concept of the simplest trajectory. The simplest trajectory is a sequential combination of the few simplest parts. These simplest parts belong to the neighbour surfaces $\mathbf{S}_{\mathbf{2}}$. We apply the sequential notation of the indices of simplest parts for notation symbolic characteristic of the simplest trajectory. These indices are separated by dot.

Two simplest trajectories are the same type trajectories if they consist of the same sequence of the simplest parts. Under domain of existence of the simplest trajectories of the same type we consider a part of the surface $\mathbf{S}_{\mathbf{2}}$ to which these trajectories belong.

In researching buck converter model with PWM there are 11 types of the simplest trajectories, each of them exclude the first, has similar type (Table 1). Similarity of the trajectories means that the same transition sequence of the switching elements of the equivalent scheme ( $\mathrm{K}_{0}$ and $\left.\mathrm{VD}\right)$ corresponds to these similar trajectories.

Simplest trajectories may consist of one, two or three simplest parts. Simplest trajectories 1, 2, 3, 6, 7 don't consist of the sliding parts.

Table 1. Simplest trajectories of the buck converter model

\begin{tabular}{|c|l|c|c|}
\hline Type & \multicolumn{1}{|c|}{ Trajectory } & $\boldsymbol{\gamma}_{0}$ & $\boldsymbol{\gamma}_{\mathbf{1}}$ \\
\hline 1 & $2(1) 2(1)$ & 1 & 0 \\
\hline 2 & $2(1) 1.12(1)$ & $\mathrm{z}_{1}$ & $1-\mathrm{z}_{1}$ \\
3 & $2(1) 1.12(2)$ & $\mathrm{z}_{1}$ & $1-\mathrm{z}_{1}$ \\
\hline 4 & $2(1) 1.13 .32(1)$ & $\mathrm{z}_{1}$ & $\mathrm{z}_{2}$ \\
5 & $2(1) 1.13 .33$ & $\mathrm{z}_{1}$ & $\mathrm{z}_{2}$ \\
\hline 6 & $2(2) 2(2)$ & 0 & 1 \\
7 & $2(2) 2(1)$ & 0 & 1 \\
\hline 8 & $2(2) 3.32(1)$ & 0 & $\mathrm{z}_{2}$ \\
9 & $2(2) 3.33$ & 0 & $\mathrm{z}_{2}$ \\
\hline 10 & $32(1)$ & 0 & 0 \\
11 & 33 & 0 & 0 \\
\hline
\end{tabular}

Here $\mathrm{z}_{1}, \mathrm{z}_{2}-$ random real values on the interval $[0 ; 1]$.

If some the simplest trajectory (next) is continuation of another simplest trajectory (previous) and taking into the consideration that state variables can't change discontinuously in the model (2-6), symbolic characteristic of the next simplest trajectory starts from the symbol which had been the last symbol of the previous symbolic 
characteristic. On the base of this rule it is possible to composite symbolic characteristics of any dynamic mode.

The simplest trajectories possible transitions structure is shown in Fig. 3. In Fig. 3 simplest trajectories are marked by circles and the possible transitions of simplest trajectories are marked by curves with arrows.

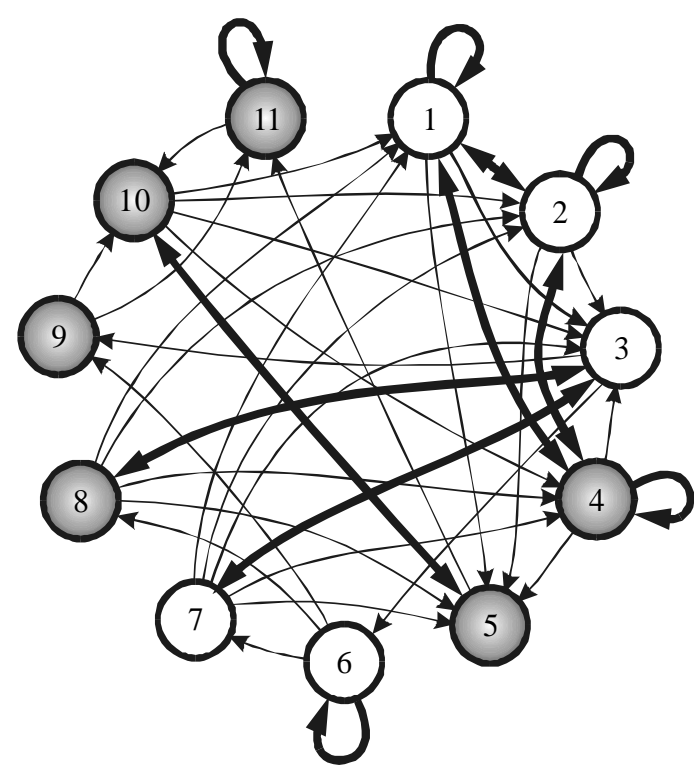

Fig. 3 - Graph of simplest trajectories transitions.

Simplest trajectories which contain sliding parts showed with shadowed circles.

The closed route in graph Fig. 3 which consists of simplest trajectories and contains $m$ vertexes corresponds to buck converter stationary mode with period $m \mathrm{~T}$. It is necessary to take into account that the existence of the closed route of the graph of simplest trajectories transitions is a necessary but not sufficient condition of stationary periodic mode existence.

The transitions which correspond to potentially possible modes with periods $1 \mathrm{~T}, 2 \mathrm{~T}$ and equilibrium points are showed in Fig. 3 by thick arrows. Symbolic characteristics of the potentially possible modes with period-2T are the following: 1,$1 ; 2,2$; 4,$4 ; 6,6 ; 11,11 ; 1,2 ; 1,4 ; 2,4 ; 3,7 ; 3,8 ; 5,10$. It is possible to composite symbolic characteristics of the potentially possible modes with high periods by means of determination of the closed route of the graph of simplest trajectories transitions.

\section{EXAMPLE OF THE BUCK CONVERTER DYNAMICS SYMBOLIC DESCRIPTION}

There is a notation of the buck converter bifurcation boundaries:
B1 - border crossing bifurcation;

B2 - period doubling border crossing bifurcation;

B3 - border collision pair bifurcation;

B4 - period doubling N-bifurcation;

B5 - saddle-node bifurcation;

B6 - period doubling and chaos border crossing bifurcation;

B7 - border collision pair bifurcation (possibly).

Subharmonic modes $m \mathrm{~T}$ are denoted by symbol $\Pi_{m . j}\left(a_{1}, a_{2}, \ldots a_{n}\right)$. Indices $m, j$ are separated by dot. Index $m$ is a frequency multiplicity of the subharmonic mode to the PWM clock instant, index $j$ is used to distinguish subharmonic modes with the same period. An expression between the brackets $\left(a_{1}, a_{2}, \ldots a_{n}\right)$ is a symbolic characteristic of any mode where $a_{1}, a_{2}, \ldots a_{n}$ are types of trajectories in Table 1. Symbol $\Pi$ with underlining denotes an instability of the respective mode. Bifurcation boundaries are denoted by $\Gamma$ symbol and by value that denotes boundary number.

A two-dimensional (2-D) piecewise smooth map of the buck converter dynamic modes is shown in Fig. 4. This 2-D map is observed by load impedance $R_{3}$ from 2 to $50 \Omega$ variation and by proportional gain $\alpha$ from 2 to 27 variation. The numerical values of the model (2-6) parameters are the following: $R_{1}=$ $0,1 \Omega ; R_{2}=0,1 \Omega ; L=10^{-4} \mathrm{H} ; C=10^{-5} \mathrm{~F} ; E=24 \mathrm{~V}$; $\beta=1 ; U_{0}=3 \mathrm{~V} ; U_{\text {ref }}=12 \mathrm{~V} ; f=10^{5} \mathrm{~Hz}$.

The description of the bifurcation boundaries on which synchronous modes disappear or become unstable is tabulated in Table 2.

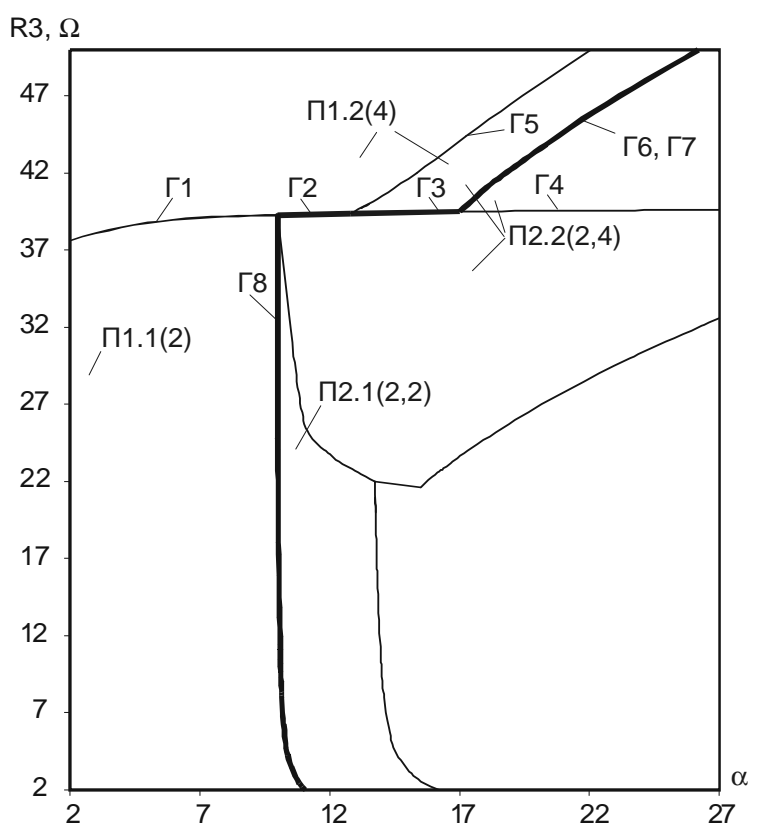

Fig. 4 - A 2-D piecewise smooth map of the buck converter dynamic modes. 
Let us demonstrate some examples of bifurcation boundary notation reading (from Table 2).

For example, let us consider the second string of Table 2. Increasing of the load impedance R3 leads to the stable synchronous mode $\Pi 1.1$ with symbolic characteristic 2 (Table 1) transits to the stable synchronous mode $\Pi 1.2$ with symbolic characteristic 4 (Table 1) at the $\Gamma 1$ boundary (Fig. 4) due to border crossing bifurcation. Symbolic characteristic 2 (Table 1) means that $\mathrm{K}_{0}$ switch is in conducting state and diode VD is in non-conducting state at the beginning of the PWM clock instant.

Table 2. The buck converter bifurcation boundaries description

\begin{tabular}{|c|c|c|c|}
\hline$\dot{\mathbf{z}}$ & 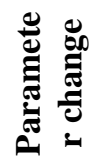 & Bifurcation transition & 苞 \\
\hline$\Gamma 1$ & $\mathrm{R} 3 \uparrow$ & $\Pi 1.1(2) \rightarrow \Pi 1.2(4)$ & B1 \\
\hline$\Gamma 2$ & $\mathrm{R} 3 \downarrow$ & $\Pi 1.2(4) \rightarrow \Pi 1.1(2)+\Pi 2.2(2,4)$ & $\mathrm{B} 2$ \\
\hline$\Gamma 3$ & $\mathrm{R} 3 \uparrow$ & $\underline{\Pi 1} .1(2) \rightarrow \bar{\Pi} 1.2(4)+\Pi 2.3(2,4)$ & B2 \\
\hline$\Gamma 4$ & $\alpha \uparrow$ & $\underline{\Pi} 1.1(2) \rightarrow \Pi 1.2(4)$ & B1 \\
\hline$\Gamma 7$ & $\alpha \downarrow$ & $\bar{\Pi} 1.2(4) \rightarrow \Pi 2.4(4,4)+\Pi 1.2(4)$ & B4 \\
\hline$\Gamma 8$ & $\alpha \uparrow$ & $\Pi 1.1(2) \rightarrow \underline{\Pi 1.1(2)+\Pi 2.1(2,2)}$ & B4 \\
\hline
\end{tabular}

$\mathrm{K}_{0}$ switch transits to non-conducting state and diode VD - to conducting state at $\gamma_{0} \mathrm{~T}$ within PWM clock instant. During $\left(1-\gamma_{0}\right) \mathrm{T}$ within PWM clock instant state of the switches is still unchangeable. Symbolic characteristic 4 (Table 1) means that $\mathrm{K}_{0}$ switch is in conducting state and diode VD is in nonconducting state at the beginning of the PWM clock instant. $\mathrm{K}_{0}$ switch transits to non-conducting state and diode $\mathrm{VD}$ - to conducting state at $\gamma_{0} \mathrm{~T}$ within PWM clock instant. Diode VD transits to nonconducting state at $\gamma_{1} \mathrm{~T}$ within PWM clock instant. During $\left(1-\gamma_{0}-\gamma_{1}\right) \mathrm{T}$ within PWM clock instant state of the switches is still unchangeable.

For example, let us consider last string of Table 2. Increasing of the proportional gain $\alpha$ leads to the stable synchronous mode $\Pi 1.1$ with symbolic characteristic 2 (Table 1) losses stability and the stable subharmonic mode $\Pi 2.1$ with double period and symbolic characteristic 2,2 (Table 1) occurs at the $\Gamma 8$ boundary (Fig. 4) due to period doubling Nbifurcation.

\section{CONCLUSIONS}

In this paper we have developed symbolic models of buck converter that demonstrates it efficiency over detailed bifurcation analysis in parameter space. An application of compact notation gives important information for analysis the regularities of the dynamic processes in the buck converter. It is necessary to take into account that this symbolic models are not unique. Any researcher has a possibility to composite his own variant of similar modeling according to the peculiarities of the pulse energy conversion systems.

An application of the symbolic models for the analysis of qualitative and quantitative changes of the quasi-periodic and chaotic motions is very perspective according to authors opinion.

\section{REFERENCES}

[1] Feigin M.I. Forced oscillations of the systems with nonlinear discontinuities. Nauka (Science). Moscow, 1994. p. 288.

[2] Bowman R. The methods of symbolic dynamics. Mir (Peace). Moscow, 1979. p. 246.

[3] Utkin V.I. Sliding modes in problems of optimization and control. Nauka (Science). Fizmatgiz (State press of physics-mathematical literature). Moscow, 1981. p. 368.

[4] Kolokolov Yu.V., Koschinsky S.L. On the Bifurcation of Stationary Motions in Impulse Systems of Automatic Control. Automation and Remote Control, no. 5, pp. 185-189, May 2000.

[5] Banerjee S., Karthik M.S., Yuan G., Yorke J.A. Bifurcations in One-Dimensional Piecewise Smooth Maps // IEEE Transactions on Circuits and Systems 1 - Theory and Applications in Switching Circuits, vol. 47, no. 3, pp. 633-643, March 2000.

[6] Banerjee S., Ott E., Yorke J.A., Yuan G.N. Anomalous bifurcations in $\mathrm{dc}-\mathrm{dc}$ converters: borderline collisions in piecewise smooth maps // Proc. IEEE Power Electronics Specialists' Conf., pp. 1337-1344, 1997.

[7] Banerjee S., Ranjan P., Grebogi C. Bifurcations in Two-Dimensional Piecewise Smooth Maps // IEEE Transactions on Circuits and Systems - Theory and Applications in Switching Circuits, vol. 47, no. 5, pp. 633-643, may 2000.

[8] Yuan G., Banerjee S., Ott E., Yorke J.A. Border-Collision Bifurcations in the Buck Converter // IEEE Transactions on Circuits and Systems 1, vol. 45, no. 7, pp. 707-716, July 1998.

[9] Tsypkin J.Z. Theory of linear pulse systems. Fizmatgiz (State press of physics-mathematical literature). Moscow, 1963. p. 968.

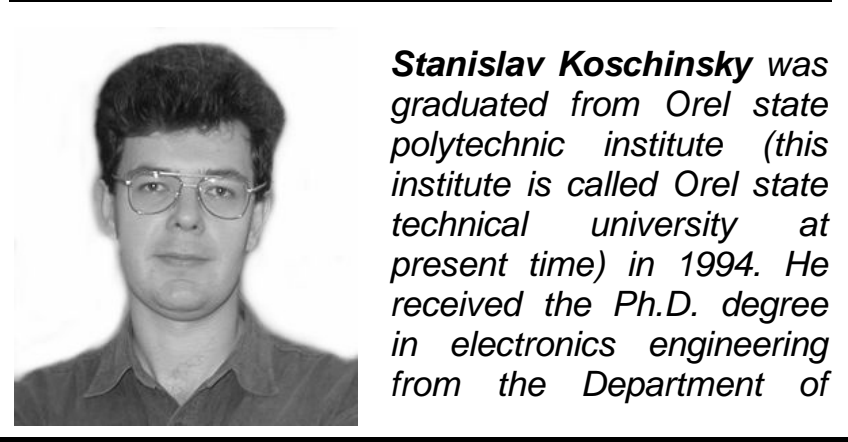


design and technology of electronic and computer systems, Orel state technical university, Orel, Russia, in 1998. Koschinsky S.L. is the associate professor of the Department of design and technology of electronic and computer systems.

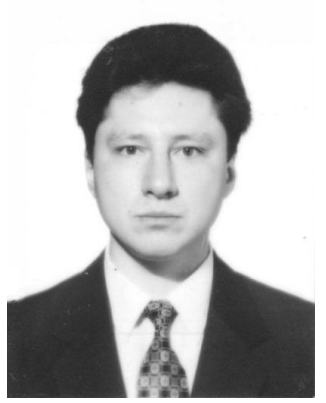

Andrey Sholonik was graduated from Orel state technical university in 1998. He received the Ph.D. degree in electronics engineering from the Department of design and technology of electronic and computer systems, Orel state technical university, Orel, Russia, in 2003.

Pavel Ustinov was entered Orel state technical university in 1999. He is a student. Ustinov P.S. takes part in the work of scientific group of the Department of design and technology of electronic and computer systems.

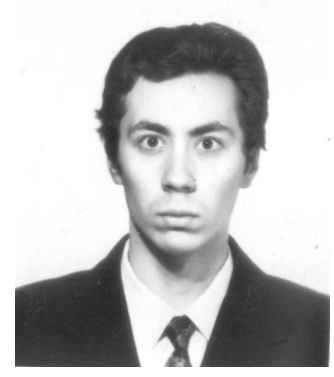

